\title{
UNA FIGURILLA OLMECA EN UN ENTIERRO DEL HORIZONTE GLÁSICO
}

\author{
Jordi Gussinyer \\ Alejandro Martínez Muriel \\ Departamento de Monumentos Prehispánicos, \\ I. N. A. H.
}

William L. Rathje y Jeremy A. Sabloff (1973:85-91), publicaron en el número anterior de Estudios de Cultura Maya (Vol. IX), el hallazgo de un interesante jade Olmeca, como ofrenda de una tumba del final del Clásico (800 d.G.) en Cozumel.

Durante las excavaciones de salvamento en el vaso de la presa La Angostura, Chiapas, encontramos un pendiente en piedra verde de estilo olmeca, como ofrenda de un entierro que probablemente se fecha al final del Periodo Clásico. Como vemos, ambas piezas fueron encontradas en condiciones muy semejantes: asociadas a entierros efectuados varios centenares de años después de la manufactura de las piezas, así como en regiones lejanas al área olmeca en la Costa del Golfo.

Rathje y Sabloff interpretan su hallazgo en función de su significación comercial, tomando en cuenta las distancias tanto temporal como espacial, para apoyar sus datos sobre el comercio "Pan-Yucateco". Creemos que este tipo de datos también tiene un significado religioso, ya que ambas piezas están asociadas a entierros de un cierto momento $\mathrm{y}$ en condiciones específicas. Por cierto, en Uaxactún también se encontró una pequeña escultura en jade de estilo olmeca, en un contexto del periodo Tzakol (Kidder, 1947: 47-48).

Desde el año de 1970 hasta 1974, la sección del salvamento del patrimonio cultural del Instituto Nacional de Antropología e Historia, con la ayuda de la Comisión Federal de Electricidad, trabajó en el salvamento arqueológico dentro del área que quedó cubierta por las aguas de la presa La Angostura en el Estado de Chiapas.

La cortina de la presa se localiza en el cañón de La Angostura 
sobre el curso superior del río Grijalva en el corazón mismo del Estado (Fig. 1).

La zona de embalse abarca una superficie aproximada de $640 \mathrm{Kms}^{2}$., y almacena un volumen de agua superior a los 18,000 millones de metros cúbicos. Las aguas cubrieron, una vez concluida la presa, más de 180 sitios arqueológicos que van desde cuevas y grupos de menos de diez montículos a centros ceromoniales en donde las estructuras se cuentan por cientos, abarcando un panorama cronológico que comprende desde el hallazgo de cerámica Ocós a la excavación de varias capillas del periodo colonial (Gussinyer, 1972a, 1976).

Los trabajos de rescate se iniciaron con un recorrido por la zona de embalse, para estructurar el salvamento, determinar los sitios arqueológicos existentes y obtener un panorama general del área que sería inundada. Se localizaron muchas zonas arqueológicas de las cuales no se tenían referencias anteriores. En este caso, se llevaron a cabo cuidadosos muestreos cerámicos, se efectuaron varios levantamientos topográficos y se elaboraron planos de algunas estructuras en los lugares que era necesario. De los sitios que ya se tenía conocimiento de su existencia se realizó una profunda investigación bibliográfica.

Después de un año de estudios de gabinete con el material del recorrido y el de la investigación, empezaron los trabajos de campo que se dividieron en cuatro temporadas. Durante la primera parte de la tercera temporada, por octubre de 1972, se estaba trabajando en el sitio denominado Laguna Francesa. Se trata de un sitio del horizonte Clásico en su etapa de mayor desarrollo que, por su tamaño, se coloca entre los sitios más grandes de cuantos existen en el área de la presa. Además, por los datos y los objetos obtenidos, nos inclinamos a creer que fue uno de los lugares más importantes de la región durante el horizonte Clásico. Por otra parte, es muy probable que Laguna Francesa fuera utilizado como un valioso punto de apoyo de los mayas, para la expansión de su cultura por la Depresión Central de Chiapas. Ostenta un centro ceremonial muy extenso con una gran cantidad de estructuras enmarcando plazas y patios. Entre ellas se destaca la impresionante mole arquitectónica de la "Acrópolis", con restos de arquitectura de franca filiación maya, influencia todavía más claramente perceptible en la cerámica obtenida, ya sea por medio de los tiestos de escombro o a través de las piezas colocadas como ofrenda, en diversas construcciones (Figs. 2 y 3 ).

Sin embargo, parece ser que él sitio arqueológico que en la ac- 
tualidad conocemos con este nombre tuvo su origen dentro del horizonte Preclásico, puesto que existe una pequeña zona llamada "La Huerta" muy cercana al centro ceremonial de época Clásica, que presenta bastantes restos de aquel horizonte (Brockington, 1959). Además, durante las excavaciones de rescate que se realizaron en el centro ceremonial se encontró dentro del relleno que formaba el núcleo de una buena parte de las estructuras exploradas una gran cantidad de tiestos del Preclásico, lo cual, ciertamente, nos confirma la existencia de un asentamiento cercano que debe haber pertenecido a este horizonte.

Entre las diversas plazas y estructuras que se eligieron para excavar, se escogió una unidad junto a la "Acrópolis" que aparentemente ofrecía un cierto interés arqueológico por la afinidad de sus componentes.

Estaba integrada por un conjunto de tres estructuras que cerraban por tres lados un patio bien trazado y claramente definido (Figs. No. 3,4 y 5 ), que formaba parte a su vez de una gran plaza; ésta en el transcurso del tiempo fue subdividiéndose en pequeñas plazas o patios, uno de los cuales es el que estamos describiendo. Por el lado Este, el patio se limitaba con un curioso edificio en forma de " $\mathrm{T}$ "; la parte Oeste quedaba abierta y por los dos lados restantes se cerraba con dos estructuras de forma muy semejante. El patio se localizaba entre la "Acrópolis" y el juego de pelota.

La primera estructura que empezó a explorarse fue la que quedaba junto al patio por el lado Sur, la número 43 (Figs. 3, 4 y 5). En realidad, se trataba de un edificio pequeño compuesto de tres cuerpos escalonados, con recubrimiento de piedra toscamente labrada para los muros de contención y un piso de estuco en la parte superior que podría corresponder por su localización a una subestructura (Figs. 7 y 8). Ua escalinata, casi imperceptible, no arrancaza del interior del patio como sería lo natural, sino que, por el contrario, por el lado exterior alcanzaba la parte alta en donde estuvo seguramente construido el templo propiamente dicho. La estructura sufrió a lo largo de su existencia varios cambios o tuvo diversas etapas constructivas que modificaron la idea primitiva, y fue, quizá, en una de ellas, cuando se construyó la escalinata con acceso por el exterior. El patio, muy bien definido, estuvo limitado por un robusto rodapié de unos $20 \mathrm{cmts}$. de alto que conformaba una banqueta de piedra sin labrar que lo rodeaba por completo (Figs. 4 y 8 ).

En la fachada posterior de la estructura No, 43 y a nivel del arranque del primer cuerpo se alzaban tres piedras burdamente labradas 
de una altura de $76 \mathrm{~cm}$. simétricamente colocadas en los extremos del edificio y en el centro del mismo, las cuales podrían corresponder por su tamaño y posición a los restos de tres pequeñas estelas lisas levantadas en este lugar (Figs. 3 y 4). En las estructuras 1A y $1 \mathrm{~B}$ de San Francisco (A-45), en la misma área de trabajo, se encontraron también tres piedras de las mismas dimensiones y colocadas en forma casi idéntica a la que acabamos de describir.

Bajo la estela central, y por debajo del arranque del primer cuerpo de la estructura, se localizó un entierro en olla, (Entierro 29), que presentaba características muy similares a otros excavados en este mismo lugar (Figs. 3 y 4). Lo que más nos llamó la atención al momento de explorarlo, fue el tamaño de la olla, puesto que se trataba de uno de los ejemplares más grandes encontrados hasta ahora en las exploraciones (Fig. 9). Junto a ella estaban colocados cuatro cajetes trípodes como ofrenda; miden alrededor de $20 \mathrm{cmts}$. de diámetro, tienen soportes cónicos y un reborde en la parte media a manera de adorno y una franja de engobe de color rojo casi anaranjado que cubría desde el borde una gran parte del interior de la vasija (Fig. 10). Se han encontrado a menudo cajetes con las mismas características, o similares, no solamente en este sitio arqueológico sino en otros lugares, y parece ser que están relacionados con ofrendas y entierros. La tapa no era otra cosa que una gran vasija embrocada sobre la olla. Tanto ésta como la tapa llevaban sencillos adornos típicos de la cerámica de su época (Fig. 9) siendo las paredes gruesas y de burda manufactura.

Unidades de este tipo, con pequeños cambios que en nada alteran el concepto general del sistema de entierro, las hemos encontrado con cierta frecuencia durante los trabajos de salvamento en esta región. Sin embargo, y esto ya nos llamó la atención, la junta ollatapadera estaba sellada por una gruesa capa de estuco a todo su alrededor, siendo la primera vez en todos los entierros excavados de esta clase que aparecía con tal detalle.

Por la forma de la olla, el tipo de enterramiento y por la cerámica asociada al entierro con sus ofrendas podría corresponder a una etapa bastante avanzada del horizonte Clásico.

En el interior de la olla se encontró un entierro secundario sumamente destruido, y entre los huesos casi deshechos una figurilla de estilo olmeca labrada en piedra (Fig. 12). A pesar de lo anómalo que parece este detalle, no es la primera vez que se encuentra una figurilla olmca relacionada con restos culturales del horizonte Clásico ya que "La pieza de Cozumel se encontró en un induable con- 
texto Clásico Tardío Maya, su estilo es incuestionablemente olmeca y por su manufactura ha sido fechada en el preclásico" (Rathje y Sabloff, 1973, p. 88).

Se conocen ya bastantes hallazgos de restos de cultura olmeca en el Estado de Chiapas, aumentando de día en día, cada vez que se llevan a cabo nuevas exploraciones. En cuanto a las esculturas de tamaño pequeño los hallazgos son también ya más frecuentes. Las piezas modeladas en barro de clara ascendencia olmeca son quizás más abundantes que las figurillas labradas en piedra. De entre estas últimas sobresale la magnífica figurilla de Ocozocuautla junto con otros restos de escultura pequeña encontrados en diferentes partes del Estado de Chiapas (Navarrete, 1971: 69-82).

La figurilla (Fig. 12) está labrada en una roca dura y mide 84 $\mathrm{mm}$. de alto y $35 \mathrm{~mm}$. de ancho. Representa a una persona de pie, completamente desnuda, sin indicación del sexo, lo cual constituye una de las características más sobresalientes de la escultura olmeca labrada en piedra (Bernal, 1968: 101). A pesar de que la pieza apareció fragmentada, sus rasgos generales corresponden a los que Miguel Covarrubias (1946: 159) reúne para caracterizar lo olmeca.

Desgraciadamente le falta la pierna derecha, el brazo derecho y el antebrazo izquierdo, este último presenta en el centro de la zona fragmentada un orificio de $3 \mathrm{~mm}$. de diámetro que podría corresponder, por su forma y disposición, a un ensamble posterior destinado a recibir la parte rota del antebrazo. Indudablemente que si la pieza se restauró, debe haber sido por su valor, por su significado y por el simbolismo religioso que representaba junto con los elementos descritos que colocan a la pequeña escultura plenamente dentro de lo olmeca; existen otros detalles, poco perceptibles, que vale la pena tomar en cuenta. De entre ellos sobresale el cabello y la forma especial de representarlo. Observando con atención las figurillas de esta índole, se nota inmediatamente que la mayor parte de ellas se muestran a menudo "... con la cabeza totalmente afeitada..." (Covarrubias, $1946 ; 160)$. Alejándose de esta particularidad, nuestra escultura exhibe una abundante cabellera índícada de una forma muy extraña y poco hábil puesto que, a partir de un círculo de unos $4 \mathrm{~mm}$. de diámetro labrado en el centro de la parte superior de la cabeza, salen de él como rayos en delgadas líneas tratando de representar el pelo, cubriendo parte de la frente y cayendo sobre las orejas perfectamente indicadas y colocadas, con sus perforaciones para recibir sendas orejeras y, al fin, deslizándose sobre la parte posterior de la cabeza la cubre por completo en líneas paralelas. Este 
rayado, para dar la sensación de la cabellera, es casi imperceptible y se llevó a cabo en una forma extraordinariamente burda, contrastando con el resto de los escasos detalles del cuerpo, que a pesar de estar sólo ligeramente marcados, son de líneas más profundas, más suaves de ejecución y sobre todo realizadas a base de curvas de grato modelado. La posición de las piernas, ligeramente fláccidas de consistencia, junto con la falta de indicaciones anatómicas en la parte posterior del cuerpo, le acercan bastante a las figurillas de estas mismas características encontradas en La Venta y zonas cercanas.

Finalmente, la pequeña escultura presenta detrás del cuello una perforación horizontal que quizás podría corresponder a una etapa de reuso, con lo cual la figurilla podría haberse utilizado por algún tiempo para llevarse colgada como pectoral o para otra intención. No sería la primera vez que piezas olmecas se han reutilizado en épocas posteriores, empleadas en algún caso para los mismos usos para los que fueron hechas o adaptadas a necesidades diferentes a su destino original (Cervantes, 1969). Existe una interesante figurilla olmeca labrada en piedra encontrada en Uaxactún, que por las características de su hallazgo y por ciertos rasgos que presenta el pequeño objeto, podría tratarse también de una pieza de reuso (Kidder, 1947: 47, 46, Figs. 37t, 74). Sin embargo, por su frontalidad, por su tamaño y por la falta de indicaciones anatómicas en la parte posterior de ella, la perforación aludida podría corresponder a la época en que se labró la escultura.

Después de un análisis de la escultura en los laboratorios de Geología del I.N.A.H. nos proporcionaron los siguientes resultados, que consideramos útiles para aspectos de identificación y procedencia de materiales de comercio:

"Estudio Petrográfico de una lámina delgada de una figurilla Olmeca de La Angostura, Chiapas".

L. 4152

M. Reyes Cortés

Aspecto Megascópico

Color verde claro y blanco.

Estructura cristalina afanítica.

Estudio Microscópico

Textura: Cristalina Subautomórfica.

Mineralogía: Albita, Magnetita, Sericita, Minerales Arcillosos, Limonita.

Origen: Extrusivo o Hipabisal de poca profundidad asociado a provincias de rocas básico-metamórfica. 
Notas: 1. - La albita está alternándose en parte y es el mineral más abundante de la muestra.

Julio 1973

Desde el punto de vista arqueológico, resulta sumamente interesante el haber encontrado dentro de una olla-entierro del horizonte Clásico una figurilla olmeca, junto con otros pequeños detalles relacionados con ella. Se ha dicho a menudo que los objetos pequeños de fácil manejo y, como consecuencia, de cómodo transporte, pueden haber viajado grandes distancias a través de regiones muy alejadas de su lugar de origen, y en esta pieza tenemos en primer lugar una evidencia de comercio.

Por otra parte, también da lugar a una serie de consideraciones que caen dentro de los conceptos del culto a los muertos con sus consiguientes derivaciones sociales; así vemos que si la pieza hubiera sido colocada simplemente como ofrenda en un entierro en olla de época Clásica, hubiera sido acomodada junto con los cajetes, como ha ocurrido con los demás entierros de esta misma índole. Sin embargo, podría ser que la figurilla, por su importancia conceptual e incluso por su valor material, hubiera sido puesta junto a la persona enterrada.

Existe una segunda posibilidad: que la figurilla hubiera sido encontrada al planear nuevas construcciones en el centro ceremonial y se encontrara en el transcurso de las obras. Por ser un objeto extraño a su cultura adquirió un valor especial y, quizás, fue colocada no como ofrenda sino más bien, como acompañante de la persona enterrada, de acuerdo con la opinión de que las figurillas olmecas de este tipo fueron colocadas en los entierros para que guiaran al difunto en su largo peregrinar en la otra vida y lo protegieran de extraños enemigos (Toscano, 1952: 200).

Podría también haber ocurrido que la figurilla hubiera sido colocada como un adorno personal, no exenta de significado religioso.

Finalmente, se destaca todavía una posibilidad más factible: como se ha dicho al principio, existe en este sitio arqueológico una pequeña zona que še ha clasificado como perteneciente al horizonte Preclásico, localizada muy cerca al actual emplazamiento del centro ceremonial. Como consecuencia, podría haberse dado el caso de que cuando se estaba sacando material en un lugar cercano, para formar el núcleo de las nuevas estructuras del centro ceremonial, "se hubiera encontrado un entierro primario con la figurilla que lo acompañaba y, por respeto o por temor hacia los restos óseos hallados, vol- 
vieran a enterrarse junto con la figurilla dentro de una olla, como venía haciéndose en su tiempo, añadiendo una nueva y sencilla ofrenda compuesta de los cuatro cajetes descritos.

Dentro del conjunto de la estructura No. 43 el entierro ocupaba un espacio de cierta categoría. Parece ser que este lugar era escogido para enterrar personas importantes puesto que nos fue posible localizar otros entierros de estas mismas características ocupando sitios idénticos en el interior de un edificio. De entre ellos sobresale el entierro No. 26 que pudo excavarse en la estructura No. 41, que cerraba por el lado norte la misma plaza. Junto a la olla, como en el entierro que estamos estudiando, fueron colocadas en forma de ofrenda dos cajetes iguales a los que ya hemos mencionado (Fig. 10). En el interior de la olla se encontraron restos humanos muy destruidos y, entre ellos, una flauta de hueso y cinco piedras pequeñas de color casi blanco (Fig. 11). Este entierro apareció en el mismo eje del que contiene la figurilla olmeca, pero en la estructura de en frente y en condiciones muy parecidas (Fig. 3 y 4 ). Además, en el patio que limita el arranque de las grandes escalinatas que conducen a la plaza intermedia de la "Acrópolis", sobre el eje longitudinal de este conjunto, se excavó una estructura bastante importante con la fachada posterior cerrando la plaza y, sobre el eje transversal de la construcción, debajo del primer cuerpo del edificio, se descubrió otro entierro en olla en circunstancias muy parecidas a las que se acaban de describir.

Todavía quedan dos elementos relacionados con la figurilla olmeca y con el entierro que pueden arrojarnos más luz al respecto.

En primer lugar, entre los restos óseos del entierro se localizó, después de sacar los huesos y de cernir cuidadosamente el polvo del interior de la olla, una pequeña cuenta de piedra verde muy desgastada que mide aproximadamente unos nueve milímetros de diámetro. Se trata, según un análisis superficial, de una serpentina con actinolita. Conocemos por los relatos de los primeros cronistas españoles algo acerca del significado de encontrar una cuenta de color verde asociada con un entierro; así Mendieta (1945, Tomo 1: 178) y Sahagún (1956, Tomo I: 296) señala la costumbre de colocarle en la boca al difunto una cuenta verde, posiblemente como símbolo del "corazón de la tierra" (Bernal, 1968: 140). Por lo tanto, no sería nada extraño que esta cuenta de serpentina hallada entre los deshechos huesos de nuestro entierro estuviera íntimamente relacionada con todo un conjunto de conceptos y costumbres funerarias, una de las cuales sería ésta de la cuenta en el interior de la boca. Esto le 
daría a un rasgo que los cronistas encontraron entre los aztecas una antigüedad bastante considerable, y también sería un elemento más para ayudarnos a concretar el desarrollo religioso de la cultura olmeca.

Además, en algunos de los huesos del interior de la olla pudieron localizarse restos de pintura roja. El color rojo ha sido desde tiempos remotísimos un elemento de un extraordinario simbolismo dentro de las culturas de todo el mundo (Cirlot, 1969: 143). No es, pues, nada extraño que en Mesoamérica exista también esta idea que arranca, con toda seguridad, de épocas anteriores a la misma cultura olmeca. Específicamente, para el pueblo olmeca, el color rojo tuvo siempre un profundo significado relacionado íntimamente con el culto a la muerte. Sabemos que, en algunos casos, el mismo "... jade se cubrió con una capa de pintura roja, como si el artista no apreciara la belleza del material o estuviera apremiado por el simbolismo del color" (Toscano, 1952: 201). Además, conocemos por datos arqueológicos, que los olmecas pintaban, en ciertas ocasiones, los jades funerarios de color rojo (Covarrubias, 1961: 60). Los olmecas arqueológicos, cuando no se trataba de personajes importantes dentro del grupo, "Sepultaban a sus muertos directamente en el suelo, en posición extendida y con frecuencia flexionada; acompañaban el cadáver con ofrendas y lo rociaban con cinabrio o pintura roja" (Piña Chán, 1964: 47). Tenemos datos arqueológicos del uso del cinabrio o color rojo para entierros en el Altiplano desde el Preclásico Inferior (Piña Chán, 1955: 68) y, para el centro del Estado de Chiapas, a partir del Preclásico Superior (Ruz, 1968: 161).

Finalmente, después de las consideraciones presentadas, nos inclinamos por la tercera solución que proponemos al principio, cuando de. cimos que al sacar el material para la construcción de nuevos edificios del centro ceremonial, los habitantes de Laguna Francesa encontraron el entierro olmeca, el cual, con todo el respeto que merecía un hallazgo de esta índole, decidieron enterrarlo de nuevo, según el sistema que ellos acostumbraban para personas importantes, añadiendo una nueva ofrenda formada por los cajetes descritos.

Por su reciente descubrimiento en un lugar muy cercano al área a que hacemos referencia, se destaca una pequeña escultura labrada en una roca de color verde claro, de casi ocho centímetros de altura. Encontrada en las cercanías de la pequeña población de Acala, representa a una persona en posición sedente con las piernas cruzadas, apoyando el resto del cuerpo sobre ellas y con los brazos juntos colocados sobre el estómago, en una actitud que parece de meditacibn. La cabeza, aunque desgraciadamente mutilada, presenta ras- 
gos muy definidos de la cultura a la que pertenece. Lo mismo puede decirse del resto del cuerpo que muestra en forma bastante clara alguna de las más importantes características de la escultura olmeca, visibles aún en los detalles menos significativos (Fig. 13).

\section{BIBLIOGRAFIA}

Bernat, Ignacio

1968 El Mundo Olmeca, Editorial Porrúa, S. A., México, D. F. Brockington, D. L.

1959 Report an Excavations at Finca Laguna Francesa. (Mecanografiado) N.W.A.F.

Cervantes, Ma. Antonieta

1969 "Dos elementos de uso ritual en el arte Olmeca", Anales, 1967-1968, séptima época, T. I. Instituto Nacional de Antropología e Historia, México, D. F.

Cirlot, Juan-Eduardo

1969 Diccionario de Símbolos. Editorial Labor, S. A., Barcelona. Coe, Michael D.

1965 "The Olmec Style and its Distributions", Archaslogy of Southern Mesoamerica. Handbook of Middle American Indias, Vol, 3, University of Texas Press, Austin.

Covarrubias, Miguel

1946 "El arte olmeca o de la Venta". Guadernos Americanos, vol. $X X V I I I, N o$. 4, México, D. F.

1961 Arte Indígena de México y Centroamérica. Universidad Na-

GuSSINYER, JORDI cional Autónoma de México, México, D. F.

1972a "Rescate Arqueológico en la Presa de la Angostura (primera temporada)". Boletín I.N.A.H., época II, No. 1, México, D. F.

1972b "Segunda Temporada de salvamento arqueológico en la Presa de La Angostura, Chis." Revista ICACH, época 2a., No. 5-6 (23-24), Tuxtla Gutiérrez.

KIDDER, A. V.

1947 The artifacts of Uaxactum Guatemala. Publication No. 576, Carnegie Institution of Washington, Washington, D. C.

Mendieta, Fray Gerónimo de

1945 Historia Eclesiástica Indiana. Editorial Salvador Chávez Hayhoe, México, D. F.

Navarrete, Garlos

1966 "Excavaciones en la presa Nezahualcóyotl, Mal Paso, Chis"., Boletín INAH No. 24, Instituto Nacional de Antropologia e Historia, México, D. F.

1971 "Algunas piezas olmecas de Chiapas y Guatemala", Anales de Antropología, vol. VIII, UNAM, México, D. F. 
Piña Chán, Román

1955 Las culturas preclásicas de la Cuenca de México. Fondo de Cultura Económica, México, D. F.

Piña Chán, Román y Luis Covarrubias

1964 El Pueblo del Jaguar. Consejo para la planeación e instalación del Museo Nacional de Antropología, México, D. F.

Pohorilenko, Anatole

1972 "La pequeña escultura", El arte olmeca. Artes de México, No. 154, México, D. F.

Rathje, William L. y Jeremy A. Sabloff

1973 "El descubrimiento de un jade Olmeca en la Isla de Cozumel, Quintana Roo, México", Estudios de Cultura Maya, vol. IX, pp. 85-91, UNAM, México.

Ruz Lhuillier, Alberto

1968 Costumbres funerarias de los antiguos mayas. Seminario de Cultura Maya, UNAM, México, D. F.

Sahagún, Fray Bernardino de

1956 Historia general de las cosas de Nueva España. Editorial Porrúa, S. A., México, D. F.

Toscano, Salvador

1952 Arte Precolombino de México y de la América Central. Instituto de Investigaciones Estéticas, UNAM, México, D. F.

Tozzer, Alfred M.

1966 Landa's Relación de las Cosas de Yucatán, Kraus Reprint Corporation New York.

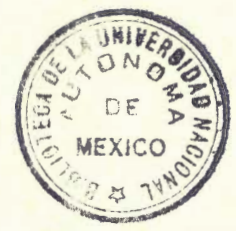

CENTRO EE ESTUDIOS

IA YAS 


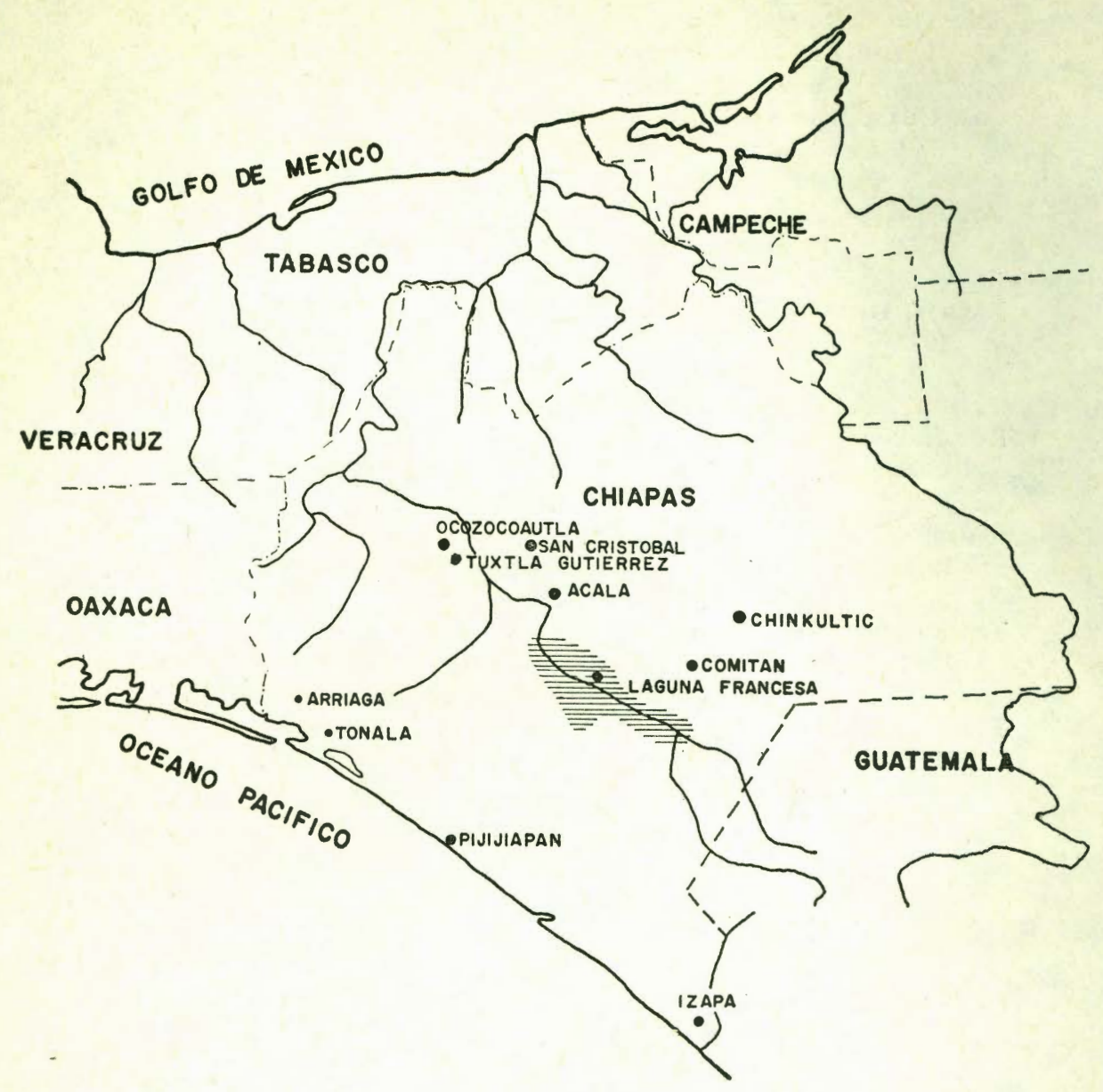

Figura 1. El Vaso de la Presa La Angostura, cubre gran parte de la De. presión Central de Chiapas; Laguna Francesa es el sitio de mayor importancia en la Región. 


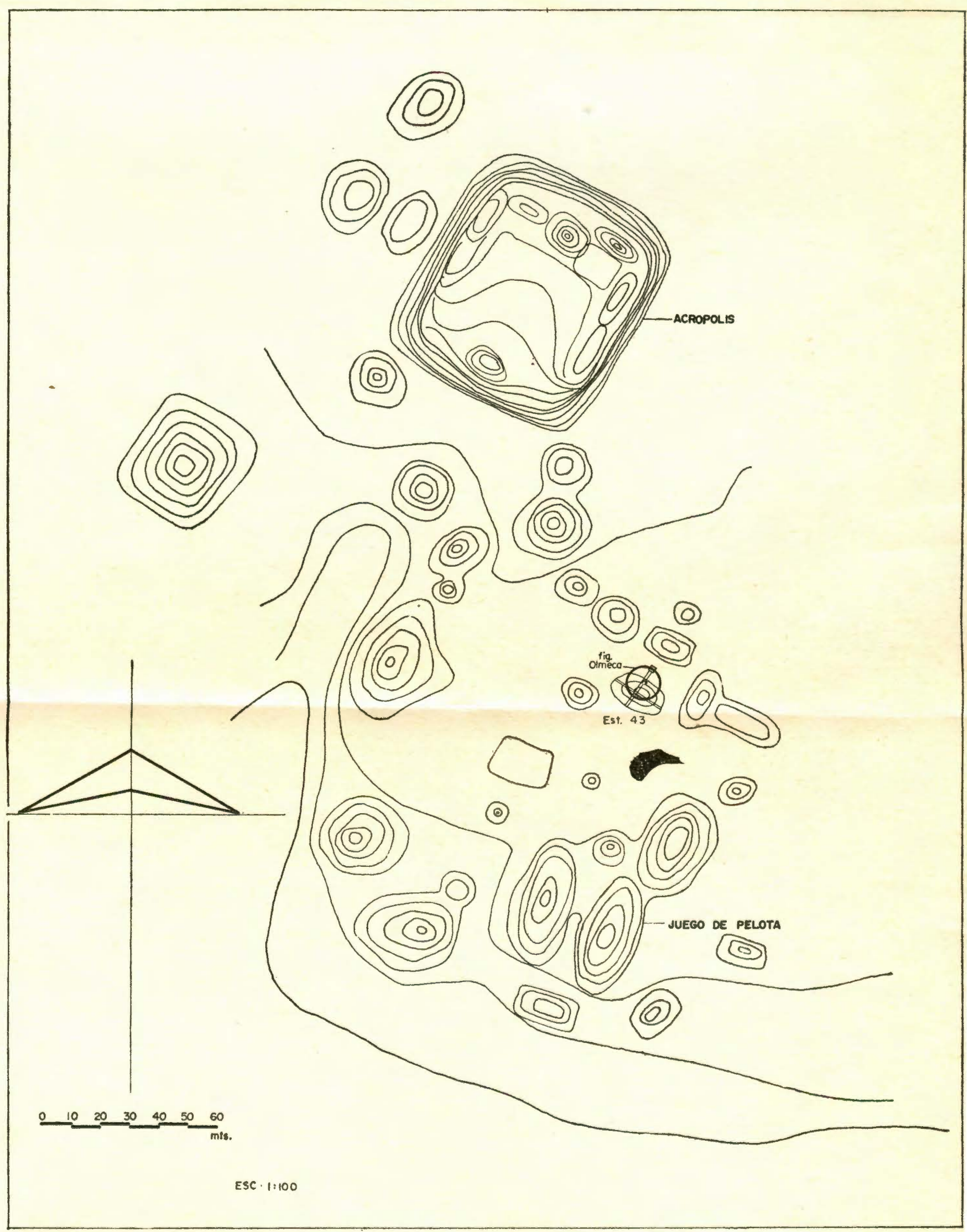

Debartamento de prebictorio SALVAMENTO DEL PATRIMONIO CULTURAL PRESA LA ANGOSTURA CHIAPAS

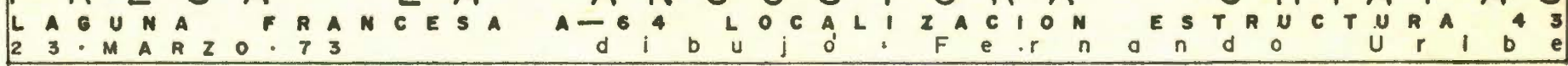




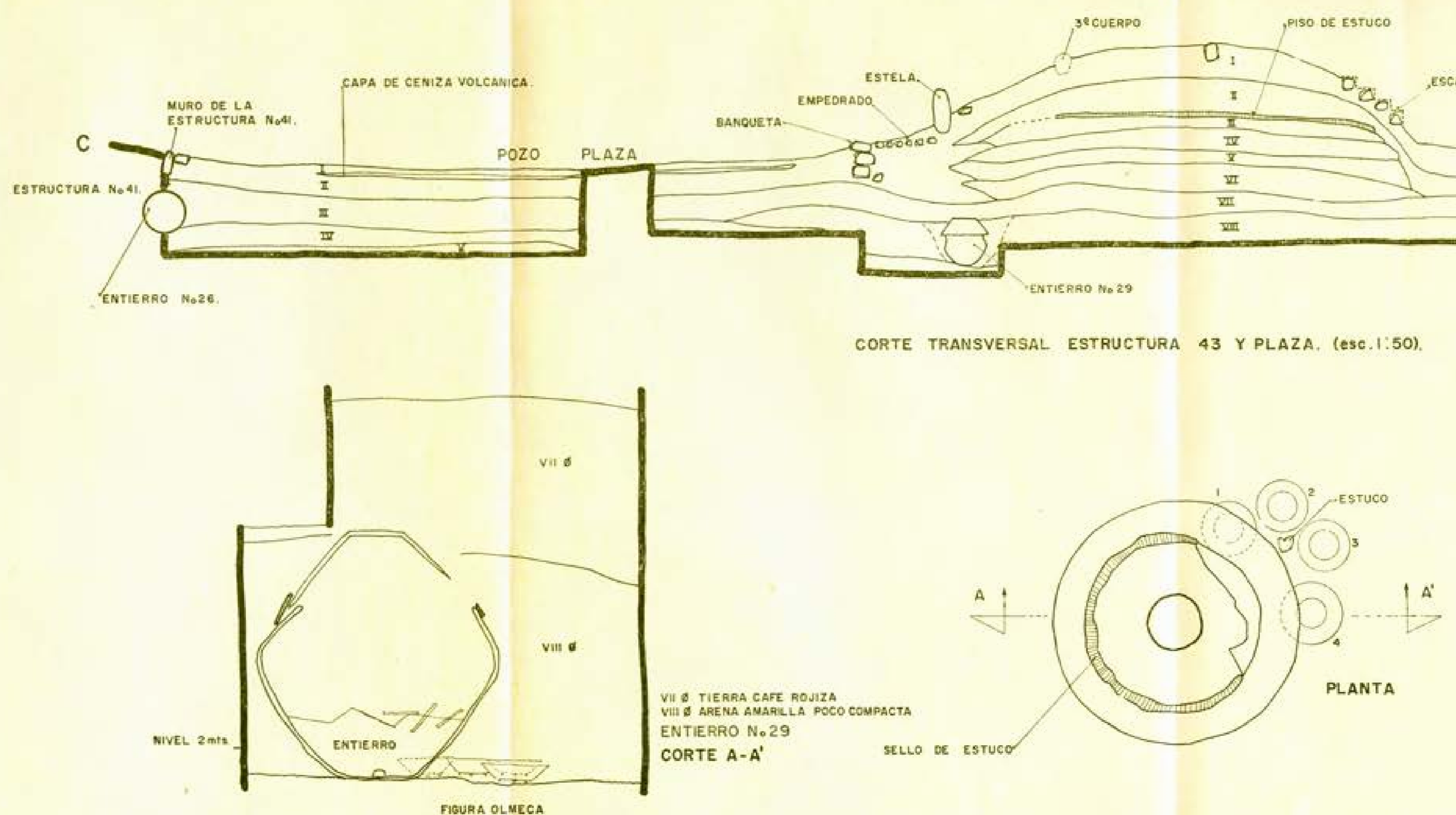

FIGURA OLMECA 


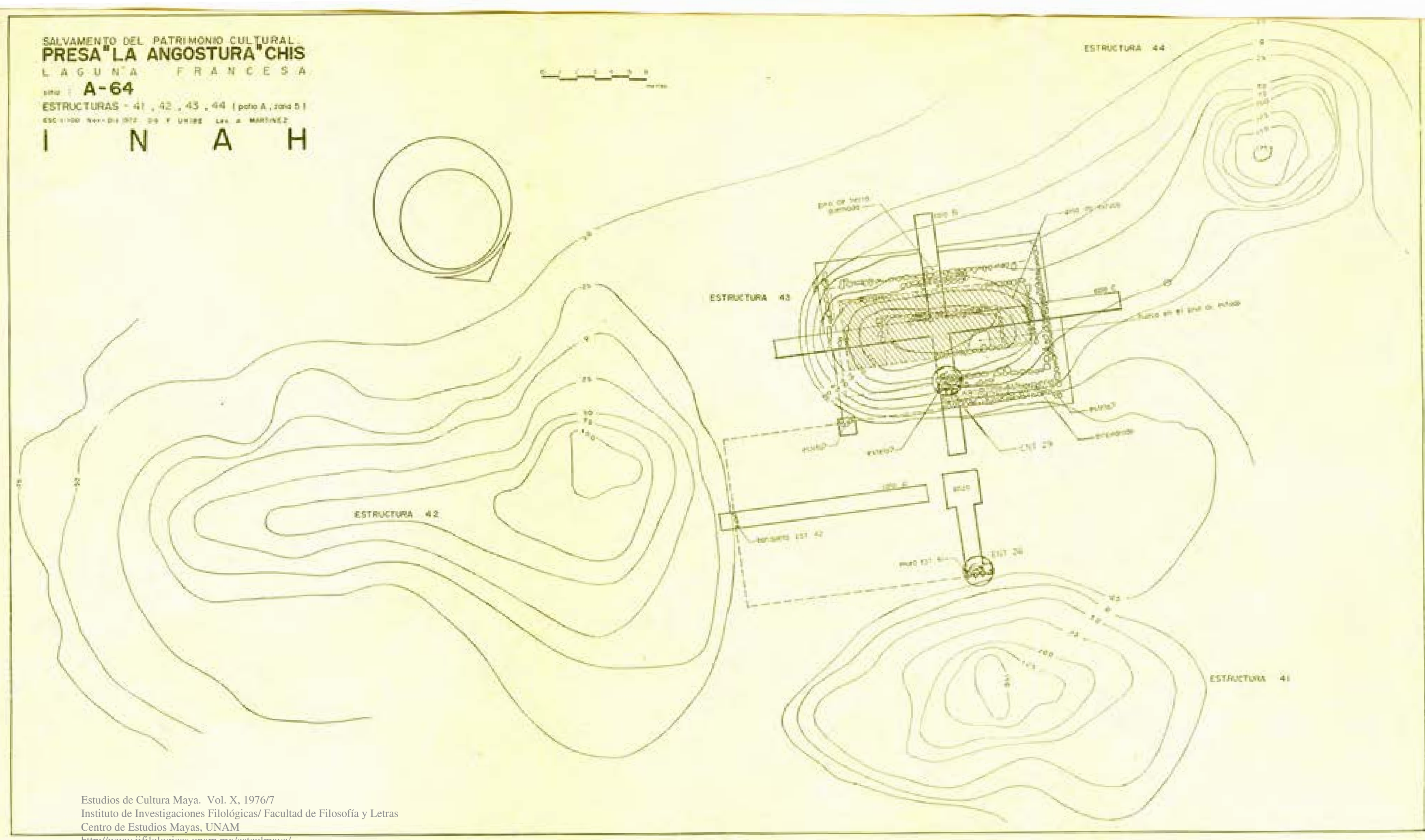




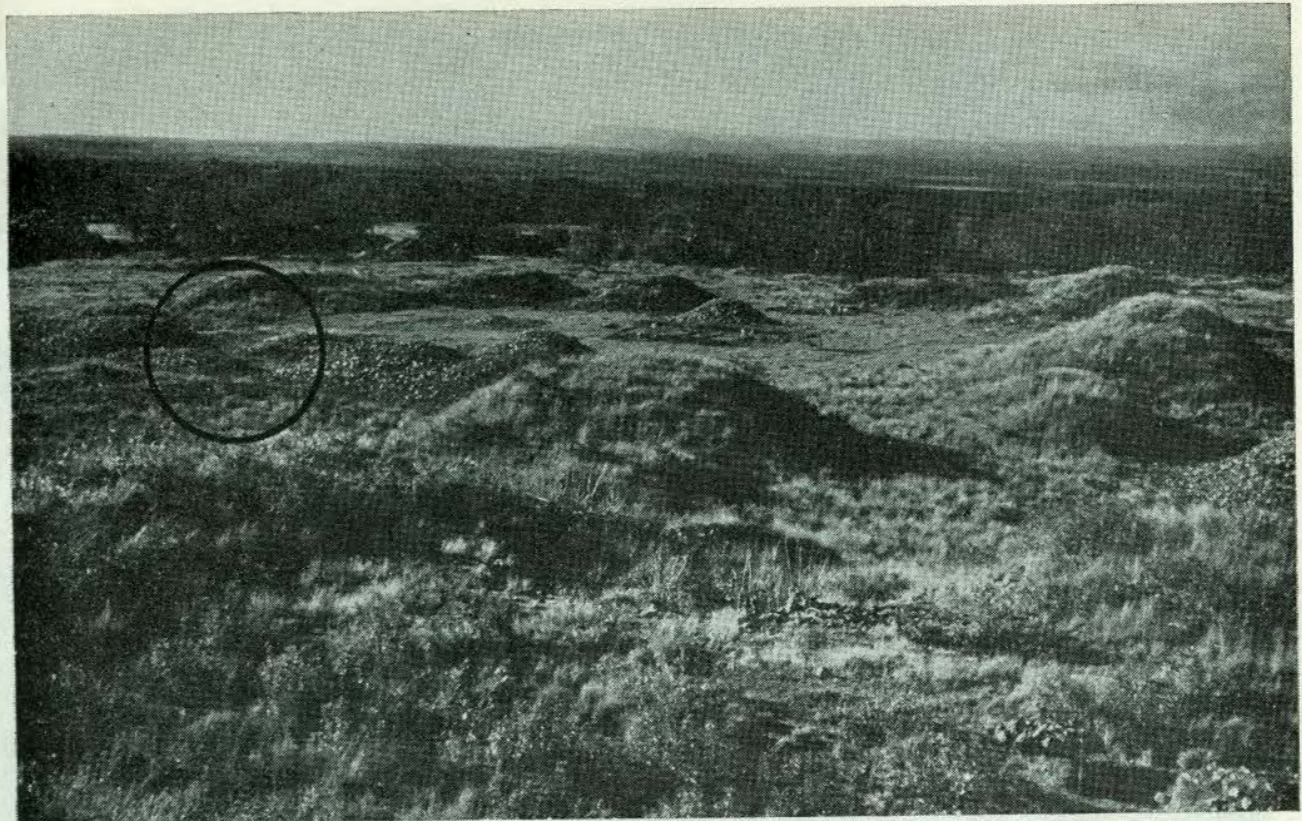

Figura. 5. Vista parcial de Laguna Francesa. Hacia el sur, al fondo, está el Río Grijalva, El círculo señala el Patio A.

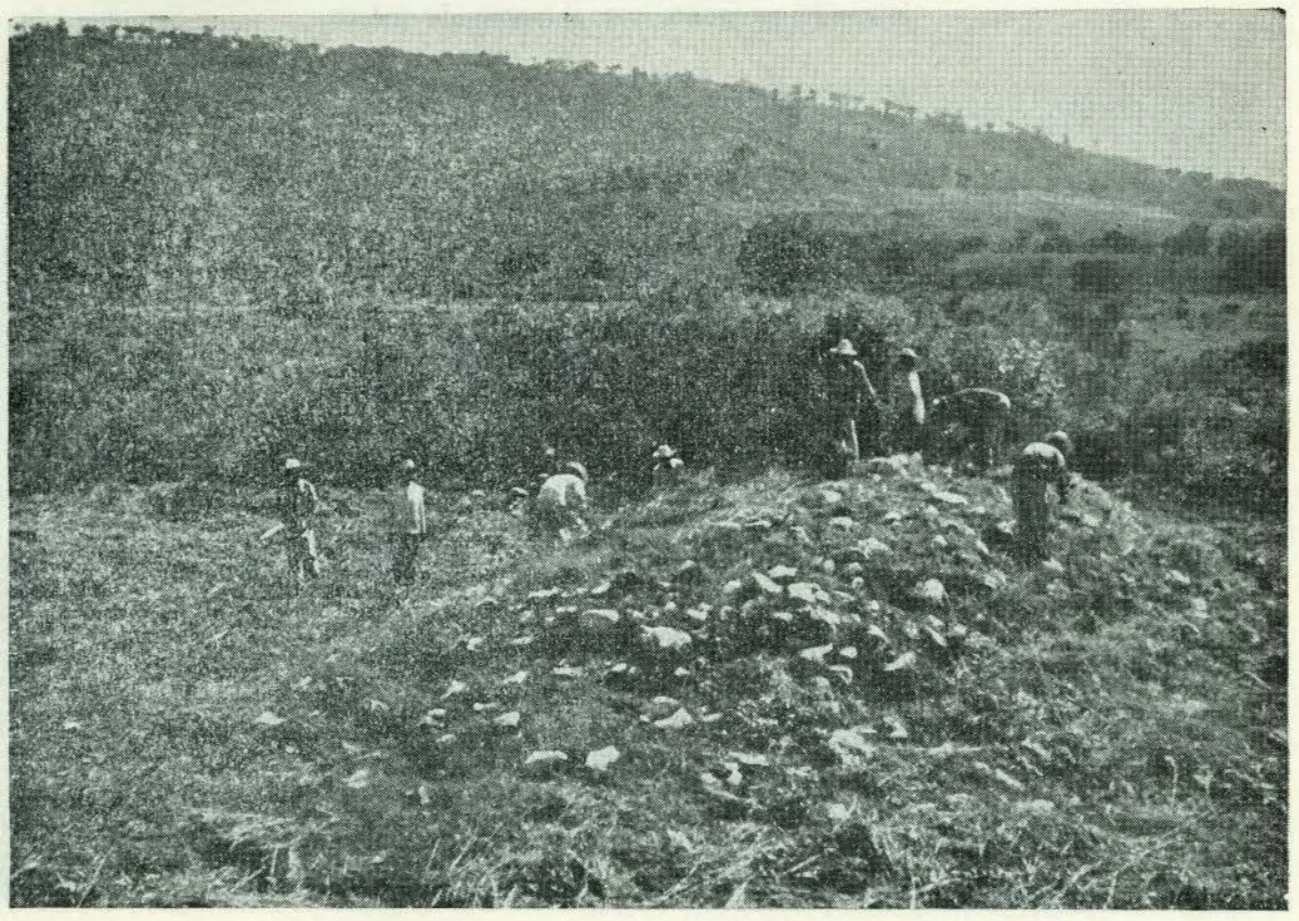

Figura. 6. Limpieza de la estructura 43, obsérvese el mal estado de conservación.

Estudios de Cultura Maya. Vol. X, 1976/7

Instituto de Investigaciones Filológicas/ Facultad de Filosofía y Letras

Centro de Estudios Mayas, UNAM

http://www.iifilologicas.unam.mx/estculmaya/ 


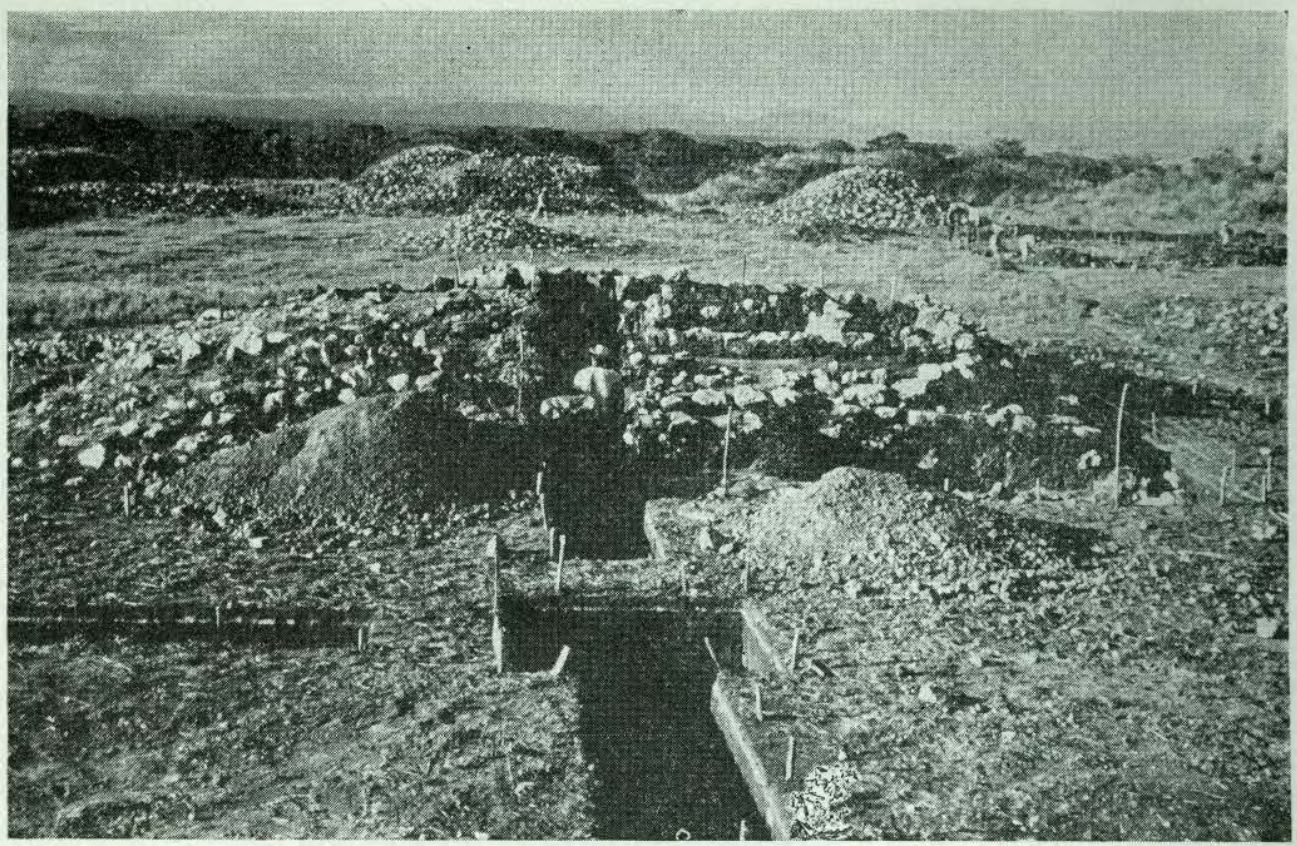

Figura. 7. Estructura No. 43 durante su excavación. En el ángulo superior izquierdo se puede ver el juego de pelota.

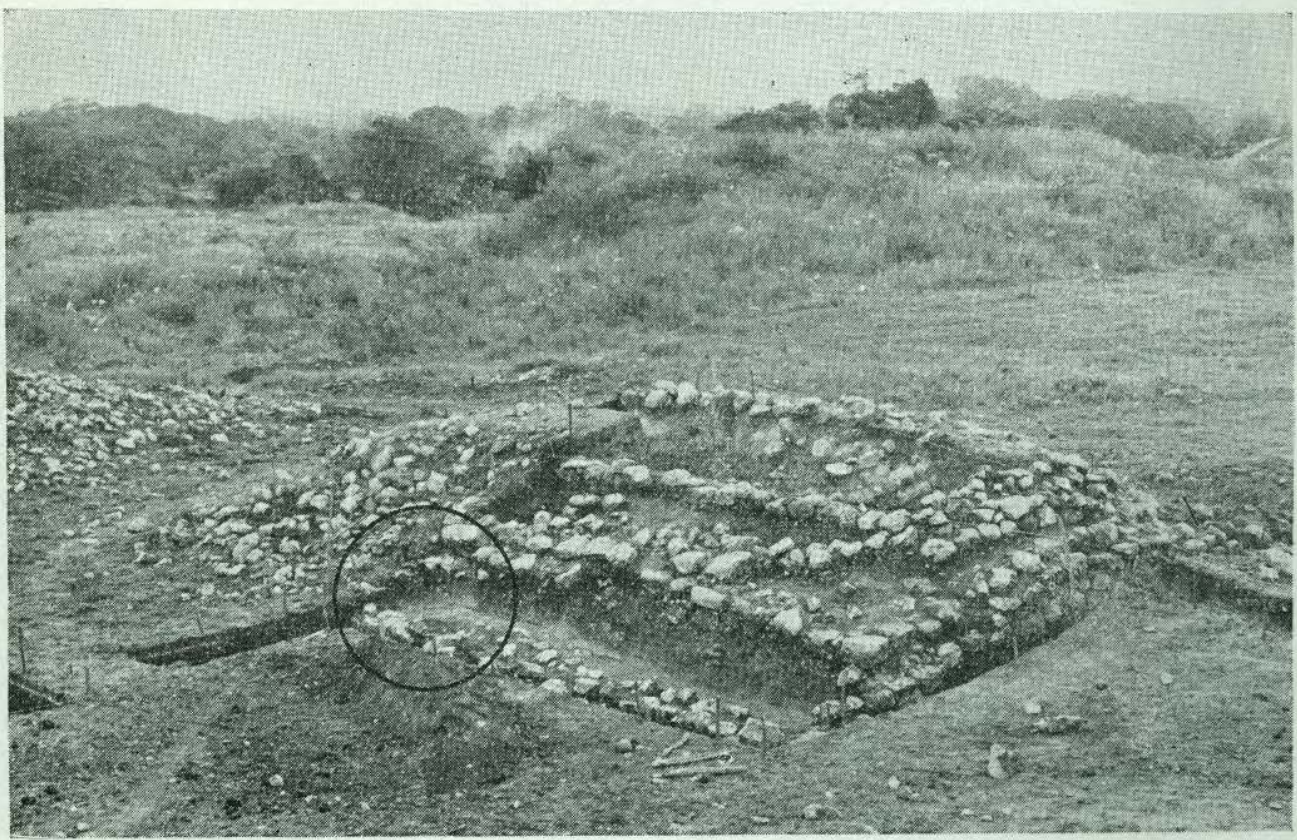

Figura. 8. Estructura 43, el círculo marca el lugar del entierro. No. 29.

Estudios de Cultura Maya. Vol. X, 1976/7

Instituto de Investigaciones Filológicas/ Facultad de Filosofía y Letras

Centro de Estudios Mayas, UNAM 


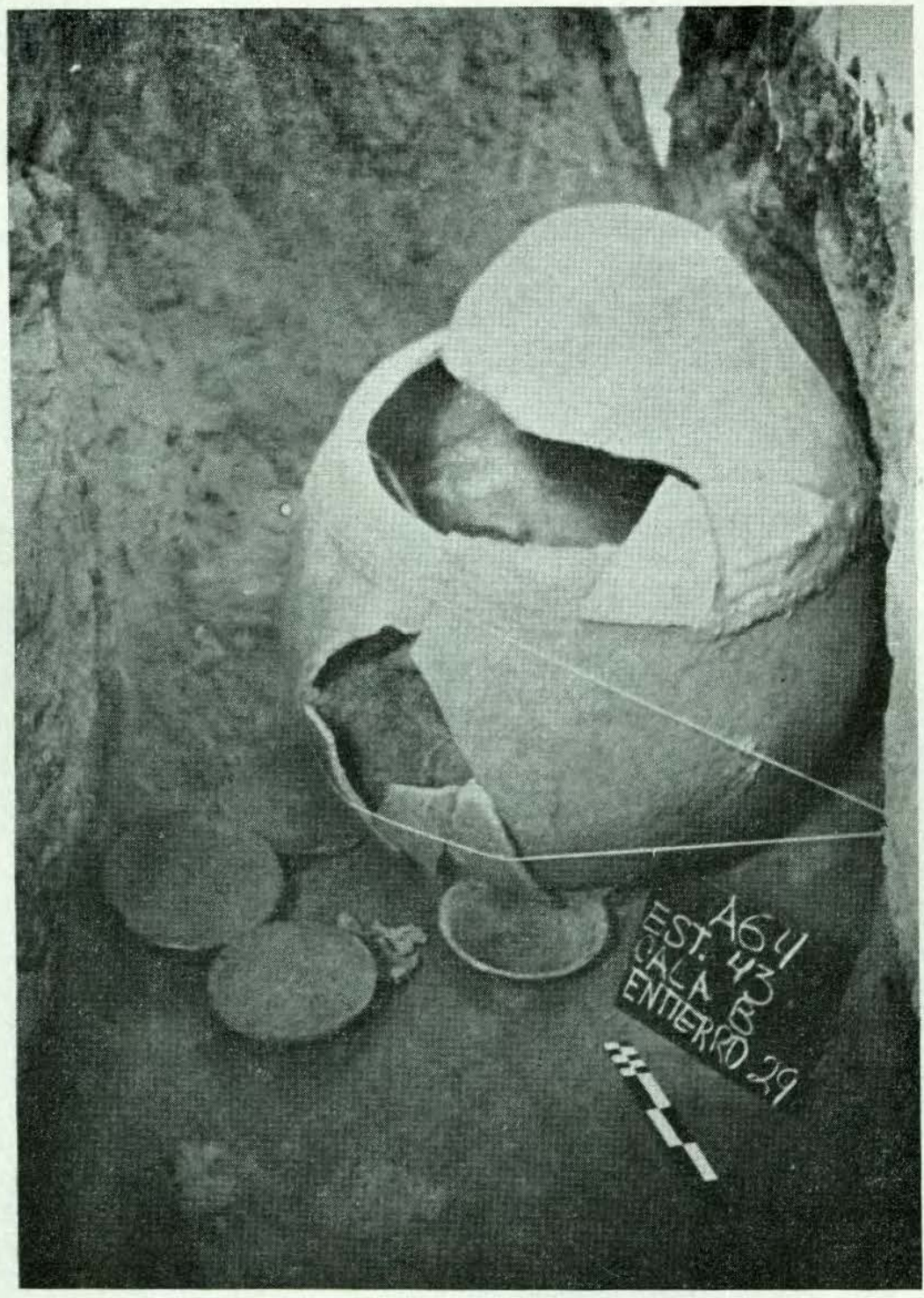

"્ّ

$\rightarrow$

ณี

包.

范

ขี

ํํํ

हี

-

동

of

ธ용

ఫั

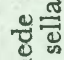

है,

号

के

을

क그

ष藏

政

표

뜸음

Estudios de Cultura Maya. Vol. X, 1976/7

Instituto de Investigaciones Filológicas/ Facultad de Filosofía y Letras Centro de Estudios Mayas, UNAM 


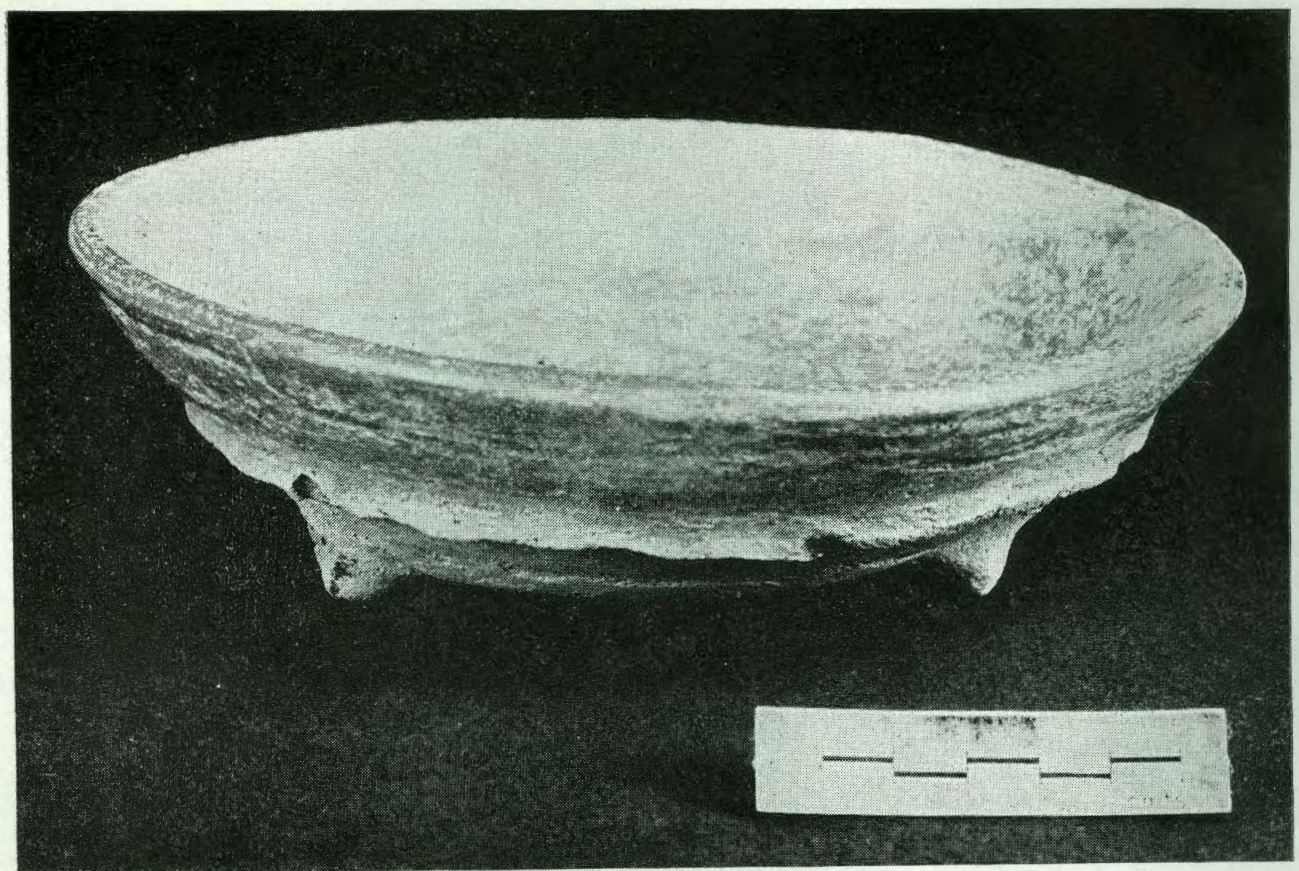

Figura, 10. Los 4 cajetes de ofrenda son de un tipo de cerámica muy común en esta región a finales del período Clásico.

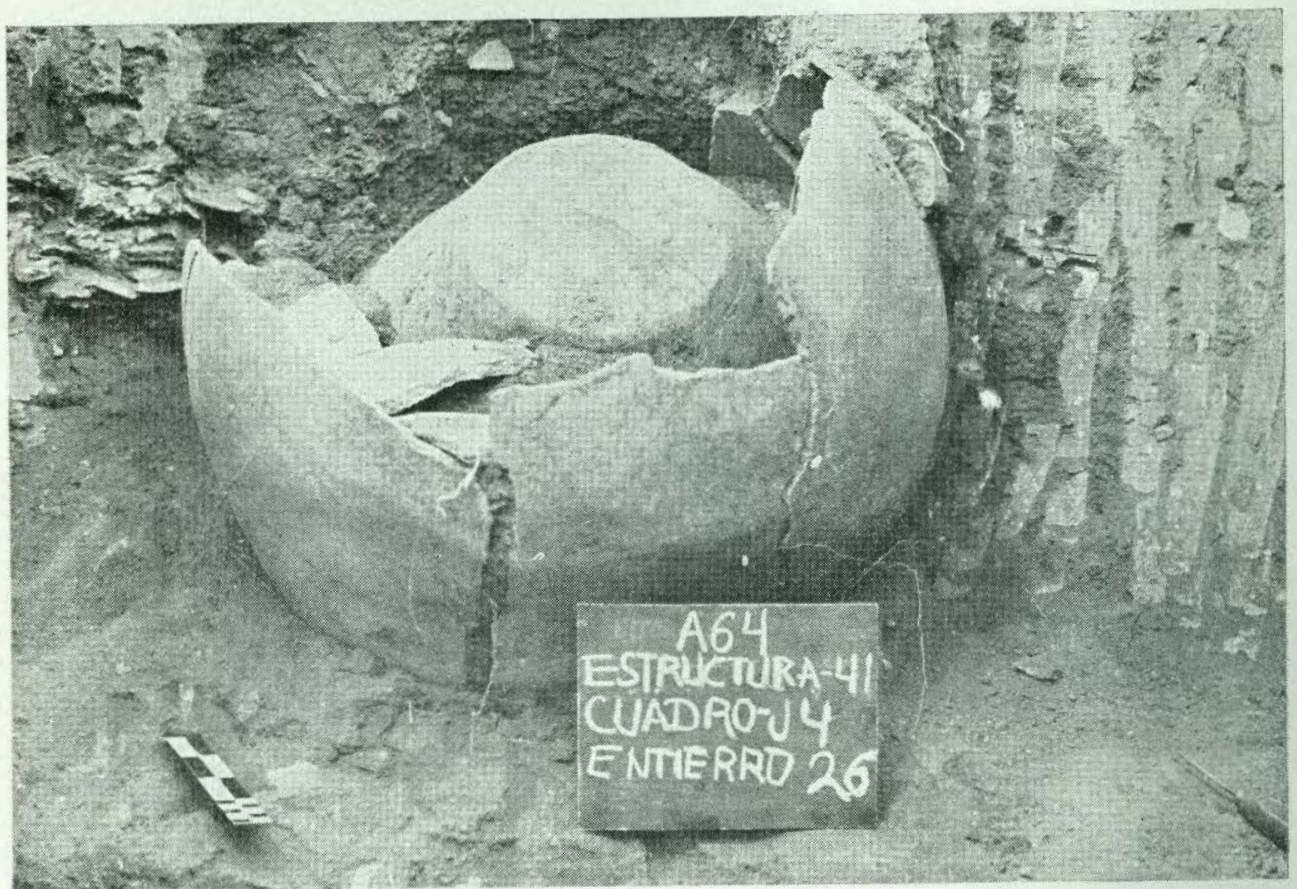

Figura. 11. Entierro No. 26 localizado en la estructura 41 frente al entierro 29; modo común de enterrar en esta región.

Estudios de Cultura Maya. Vol. X, 1976/7

Instituto de Investigaciones Filológicas/ Facultad de Filosofía y Letras

Centro de Estudios Mayas, UNAM

http://www.iifilologicas.unam.mx/estculmaya/ 


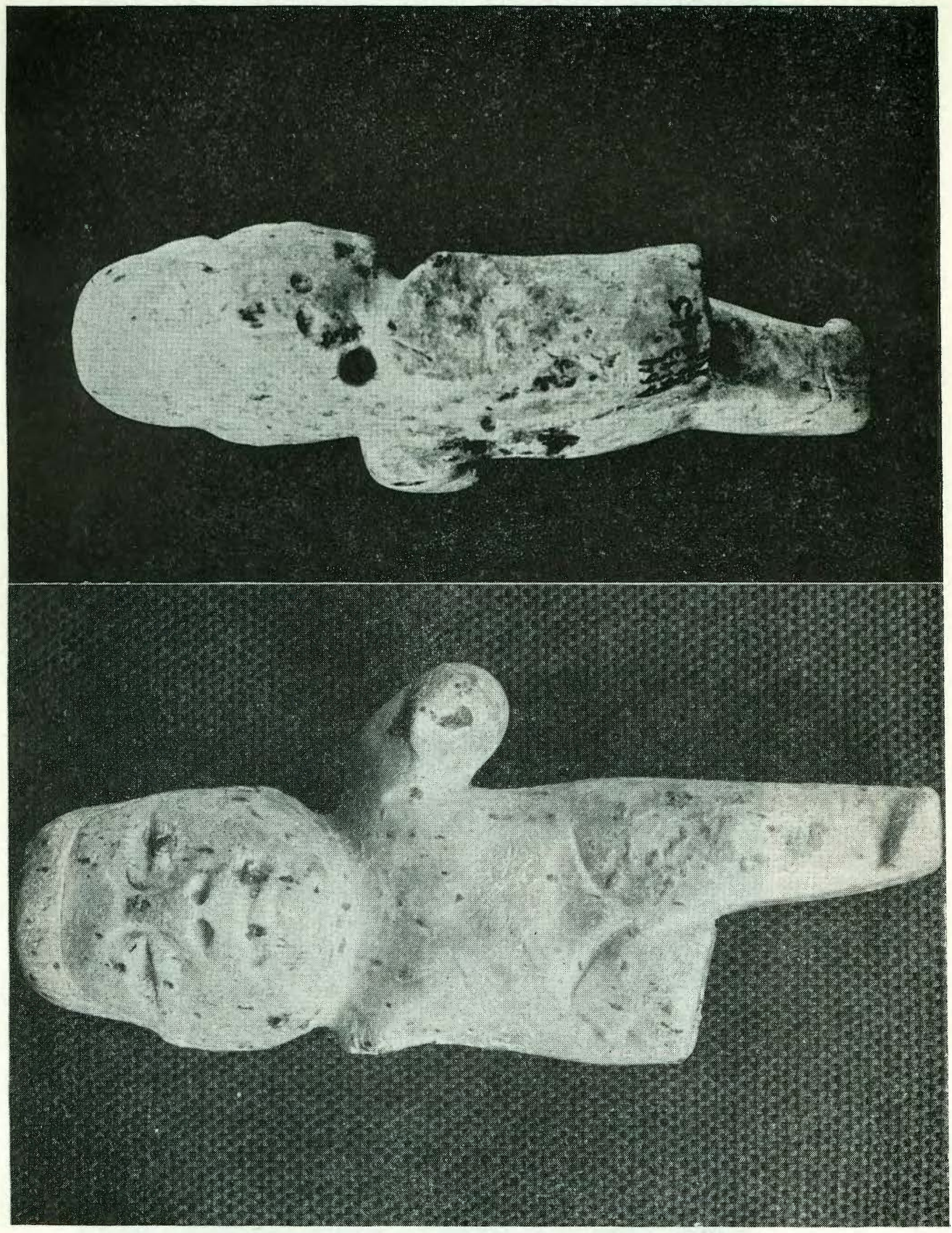

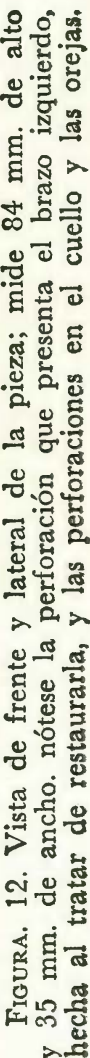




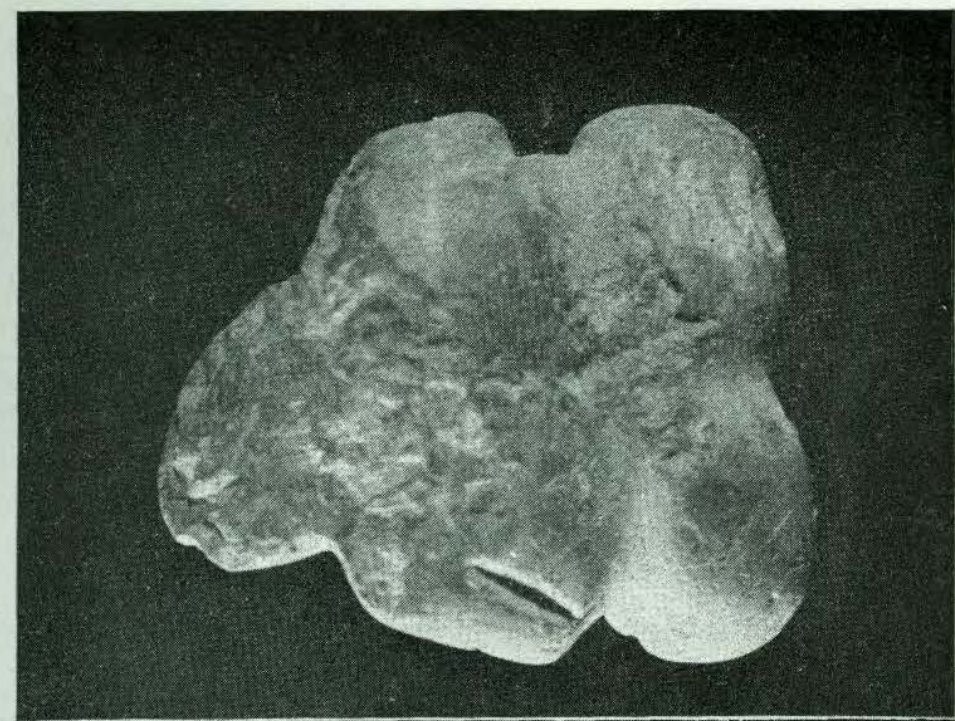

घี का

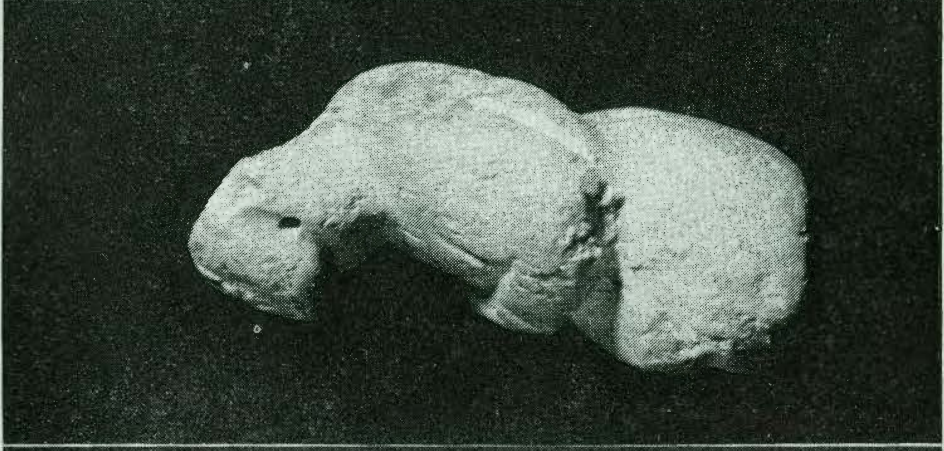

ชับ

कृำ

幽.

ง

ธี

넌엉

뜡용

ช

ํㅐ멿

똥

品

结

क्षे

造

Estudios de Cultura Maya. Vol. X, 1976/7

Instituto de Investigaciones Filológicas/ Facultad de Filosofía y Letras Centro de Estudios Mayas, UNAM 